\title{
Towards Realisation of a Non-Invasive Blood Glucose Sensor Using Microstripline
}

This paper was downloaded from TechRxiv (https://www.techrxiv.org).

\section{LICENSE}

CC BY 4.0

SUBMISSION DATE / POSTED DATE

$11-01-2021 / 13-01-2021$

\section{CITATION}

Zeising, Samuel; Kirchner, Jens; Khalili, Hossein Fazeli; Ahmed, Doaa; Lübke, Maximilian; Thalmayer, Angelika; et al. (2021): Towards Realisation of a Non-Invasive Blood Glucose Sensor Using Microstripline. TechRxiv. Preprint. https://doi.org/10.36227/techrxiv.13553528.v1

$\mathrm{DOI}$

10.36227/techrxiv.13553528.v1 


\title{
Towards Realisation of a Non-Invasive Blood Glucose Sensor Using Microstripline
}

\author{
Samuel Zeising, Jens Kirchner, Hossein Fazeli Khalili, Doaa Ahmed, Maximilian Lübke, \\ Angelika Thalmayer and Georg Fischer \\ Institute for Electronics Engineering \\ Friedrich-Alexander-University Erlangen-Nuremberg (FAU) \\ Erlangen, Germany \\ E-mail: samuel.zeising@fau.de
}

\begin{abstract}
In this paper, the design of a microwave based noninvasive glucose sensor at the operating frequency of $19 \mathrm{GHz}$ is presented. Worldwide, about $\mathbf{4 5 1}$ million adults suffer from diabetes. For optimal therapy, people with diabetes should monitor their blood glucose level continuously over the day. For that purpose non-invasive microwave based glucose sensors are under research. Non-invasive microwave based glucose sensors must automatically perform continuous measurements with a high and stable accuracy. However, there exists no reliable noninvasive microwave based glucose sensor up to now.

The proposed sensor is designed based on a two-port microstripline (MSL) network. Water with various glucose concentration from 0 to $500 \mathrm{mg} / \mathrm{dl}$ is placed in a slot between the two ports. The simulation results show that the phase change in the reflection coefficient for the various glucose concentration depends on the matching. The measurement of the glucose concentration $10 \mathrm{mg} / \mathrm{dl}$ can be realized by applying a taper ground plane as a matching technique decreasing reflections at the interface between the microstripline substrate and the water.

The proposed sensor achieves a sensitivity of $2^{\circ}$ phase change per $10 \mathrm{mg} / \mathrm{dl}$ glucose concentration variation. In the future, the simulation results should be validated in an experimental setup.
\end{abstract}

Index Terms-Diabetes, glucose sensor, non-invasive sensor, microstripline.

\section{INTRODUCTION}

Diabetes is a chronic, metabolic disease, which can cause heart disease and other organ damage over time. There are approximately 451 million adult people suffering from diabetes, circa one out of eleven adults. Furthermore, in 2017, about 5 million deaths were reported due to diabetes [1].

Normal fasting blood glucose (BG) levels are in the range of $70-110 \mathrm{mg} / \mathrm{dl}$. A slight deviation from the normal BG level can lead to a dysfunction of the energy-balance and the carbohydrate toleration [2]. Therefore, for the diagnosis and treatment of diabetes, it is imperative to measure and control the BG level accurately and frequently. BG level sensors are divided into invasive, non-invasive and minimal invasive sensors. A typical measurement tool is the invasive BG meter combined with test strips. A blood sample is taken several times a day by pricking the finger, which is inconvenient, painful and time-consuming.

To overcome these problems, non-invasive continuousglucose-monitoring (CGM) is a promising alternative [2].
Non-invasive BG-concentration sensors have increased researchers' interest, since the process is painless and applicable for CGM [3].

For that purpose, the microwave based sensors are wellestablished, since in the microwave range, the electromagnetic waves can deeply penetrate into the human tissue regions, where the main blood vessels are located and the BG level can accurately be measured [4]. The concept of a microwave based sensor is to measure the amplitude and phase variations in the scattering parameters, when dielectric properties of a material under test (MUT) change. The authors in [5]-[10] have used a resonant structure. The resonance frequency of the waveguide structure depends on a couple of parameters, one of them is the permittivity of the MUT. Any variations in the BG concentration change its dielectric properties and hence the relaxation frequency, at which the imaginary part of the relative permittivity has a maximum.

One problem of former approaches is that the operating frequencies of resonant sensors are below [5]-[9] or above [10] the relaxation frequency of their deployed MUT. Another problem is that in [5]-[8] a sub-mm gap was used, where the electric field strength is concentrated to measure the glucose concentration, since the human skin is in the mm-range it is not appropriate to use a resonator, which is based on a small gap.

In this paper, a novel microwave based non-invasive glucose sensor is proposed and discussed. The operating frequency of the proposed sensor is adjusted to the relaxation frequency of the MUT. In addition, the sensor does not depend on a small gap. The glucose concentration thus is measured and averaged across an area in the mm-range.

\section{Methods}

\section{A. Sensor Principle}

Water is a dipole composed of two dominantly positive hydrogen atoms and one dominantly negative oxygen atom (Fig. 1). Taking this into account is essential as human blood plasma consists mainly of water. The functional group hydroxyl plays an important role in the dielectric properties of an MUT. An electric dipole moment $\overrightarrow{\mathbf{p}}_{\mathrm{n}}$, given in Coulomb- 


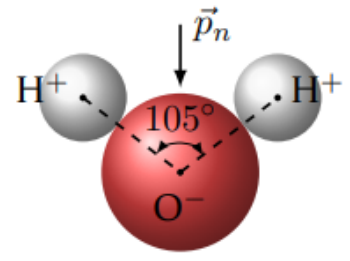

Fig. 1: The structure of a water molecule [11].

meters $(\mathrm{Cm})$, exists due to the different polarity of hydrogen and oxygen. Considering the following equations [12]:

$$
\begin{aligned}
\overrightarrow{\mathbf{p}}_{\mathrm{n}} & =\alpha_{\mathrm{pol}} \cdot \overrightarrow{\mathbf{E}}_{\mathrm{loc}} \\
\varepsilon_{\mathrm{r}, \mathrm{stat}} & =\left(\alpha_{\mathrm{pol}} \cdot n / \varepsilon_{0}\right)+1
\end{aligned}
$$

$\alpha_{\text {pol }}$ is the polarization in $\mathrm{Cm}^{2} / \mathrm{V} ; \overrightarrow{\mathbf{E}}_{\text {loc }}$ is the local electric field in $\mathrm{V} / \mathrm{m} ; \varepsilon_{\mathrm{r} \text {, stat }}$ is the relative permittivity in the steady state, which is 78.5 for water [13]; $\varepsilon_{0}$ is the permittivity in vacuum and has a value of $8.854 \times 10^{-12} \mathrm{~F} / \mathrm{m}$ and $n$ is the volume number density in $1 / \mathrm{m}^{3}$. If the polarization of a material is high, like in the case of water, the relative permittivity is high as well. In consequence, glucose which is a weak dipole and, therefore, has only a small polarization, it also has a smaller relative permittivity. Thus, the total permittivity of a $\mathrm{BG}$ solution decreases with an increased glucose concentration. The relative permittivity also depends on the applied frequency [12]. This is described by Debye's equation [14]:

$$
\begin{aligned}
& \underline{\varepsilon}_{\mathrm{r}}(\omega)=\varepsilon^{\prime}(\omega)-j \varepsilon^{\prime \prime}(\omega) \\
& \underline{\varepsilon}_{\mathrm{r}}(\omega)=\varepsilon_{\text {inf }}+\left(\varepsilon_{\mathrm{r}, \text { stat }}-\varepsilon_{\text {inf }}\right) /\left(1+j \omega \tau_{\text {relax }}\right)
\end{aligned}
$$

where $\varepsilon^{\prime}(\omega)$ and $\varepsilon^{\prime \prime}(\omega)$ are the real and imaginary part of the relative permittivity, $\tau_{\text {relax }}$ is the relaxation time, which describes the time delay of the polarization process and $\varepsilon_{\mathrm{inf}}$ is the permittivity at high frequencies. For water at $25^{\circ} \mathrm{C}, \tau_{\text {relax }}$ and $\varepsilon_{\text {inf }}$ are approximately $8.3 \mathrm{ps}$ and 5.3, respectively [13]. If an alternating electric field is applied, the polarization of the molecules can not follow instantly. If the glucose concentration is increased, $\tau_{\text {relax }}$ also increases. This results in a frequency shift of the real- and imaginary-part of $\underline{\varepsilon}_{\mathrm{r}}(\omega)$ towards lower frequencies (Fig. 2). The sensor principle is based on the measurement of the scattering parameters. If the transmission parameter $S_{21}$ of a probe is determined, there is a maximum of loss around the relaxation frequency. Therefore, if the glucose concentration is increased, there should be a frequency shift in the scattering parameters, which is visible in the amplitude and the phase.

\section{B. Design of the Sensor}

The goal of the design is to match the reflection $S_{11}$ at the relaxation frequency of water, which is around $19 \mathrm{GHz}$. In this case, the electromagnetic wave can penetrate into the water without much reflection. All simulations are done in COMSOL Multiphysics $^{\circledR}$. A microstripline (MSL) is used for the sensor approach. For frequencies around $19 \mathrm{GHz}$, a substrate with a dielectric constant of $\varepsilon_{\mathrm{r}_{\mathrm{s}}}=10.7$ is chosen. The sensor is designed as a two-port network. The MSL is adjusted for a

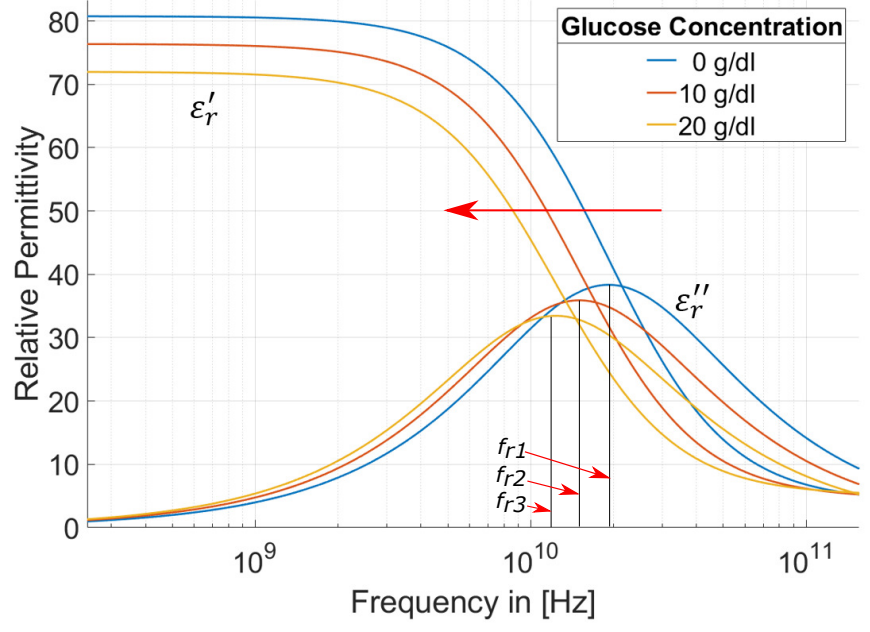

Fig. 2: The relative permittivity versus frequency. The relative permittivity and hence the relaxation frequency $f_{\mathrm{r} 1}$ is shifted towards lower frequencies $f_{\mathrm{r} 2}$ and $f_{\mathrm{r} 3}$ by increasing the glucose concentration.

characteristic impedance of $50 \Omega$. Therefore, as shown in Fig. 3 , the height of the substrate is equal to $0.4 \mathrm{~mm}$, the width of the substrate $20 \mathrm{~mm}$ and the width of the microstrip line $0.36 \mathrm{~mm}$. The upper conductor of the MSL is shown in red.

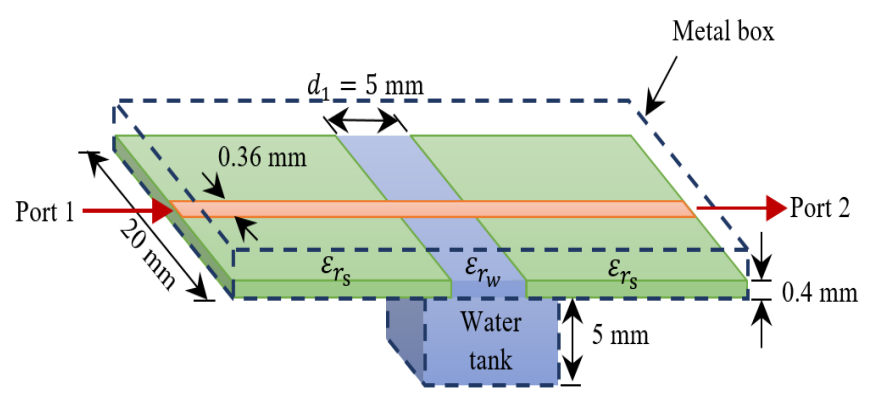

Fig. 3: The geometry of the proposed sensor. The substrate is shown in green, the upper conductor in red, the water in blue and the metal-box in dashed lines. Port 1 and Port 2 of the two-port network are identified with the red arrows.

A water tank of a depth $5 \mathrm{~mm}$ is placed under this model in a way that water fills a slot of width $d_{1}=5 \mathrm{~mm}$ between the two parts of the substrate. This water solution mimics the behaviour of the permittivity of a blood-glucose mixture in the microwave frequency range, whose dielectric properties are defined in COMSOL based on a model introduced in [15] and [16]. The slot makes it possible for electromagnetic waves to interact with the water-glucose solution underneath. To prevent electromagnetic-compatibility problems, a metal box (dashed lines) is set around the substrate and all boundaries of the water tank. A two-staged taper with the parameters $t_{1}$ and $t_{2}$ has been added (Fig. 4). The model is surrounded by an air-box. 


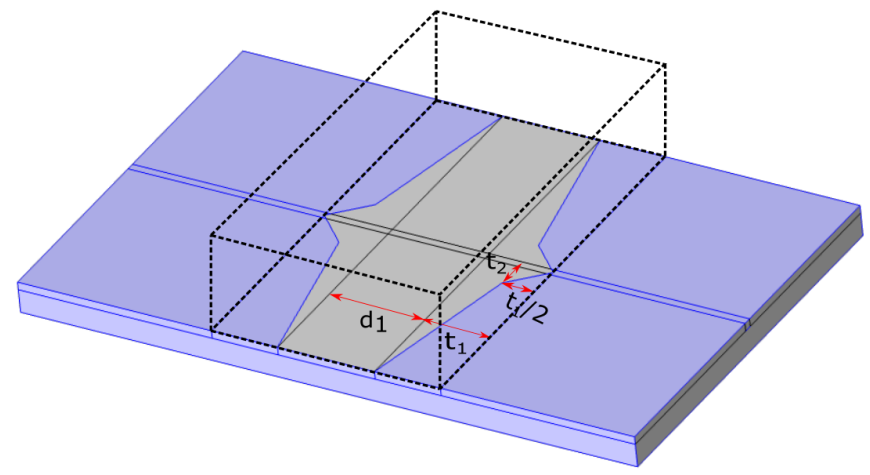

Fig. 4: The geometry of the ground plane and the metal-box (blue), the water-tank is in dashed lines: $t_{1}$ is the taper-length, $t_{2}$ is the taper-width, $d_{1}$ is the length of the water-filled slot in the substrate.

The frequency of the matching can be adjusted with the taper-length $t_{1}$. The matching can be further optimized by adjusting the taper-width with parameter $t_{2}$.

\section{RESULTS}

Inspired by the Clarke-Error-Grid [17], simulation studies are conducted with a fine step-size of $10 \mathrm{mg} / \mathrm{dl}$ from $0 \mathrm{mg} / \mathrm{dl}$ to $110 \mathrm{mg} / \mathrm{dl}$ and a coarser step-size of $50 \mathrm{mg} / \mathrm{dl}$ from $0 \mathrm{mg} / \mathrm{dl}$ to $500 \mathrm{mg} / \mathrm{dl}$. The Clarke-Error-Grid suggests a maximal deviation of $20 \%$ around $70 \mathrm{mg} / \mathrm{dl}$, which is $14 \mathrm{mg} / \mathrm{dl}$. The smallest allowed tolerance for glucose level deviations is around $70 \mathrm{mg} / \mathrm{dl}$, therefore, a step-size of $10 \mathrm{mg} / \mathrm{dl}$ in the range of $0 \mathrm{mg} / \mathrm{dl}$ to $110 \mathrm{mg} / \mathrm{dl}$ is convenient.

For all simulations, the sensor is set up with lumped ports. Fig. 5 shows port 1 and the excited fundamental mode $\mathrm{HE}_{0}$ at $19 \mathrm{GHz}$. The matching process is conducted with a glucose concentration of $0 \mathrm{mg} / \mathrm{dl}$. For a taper-length $t_{1}$ of $3.4 \mathrm{~mm}$

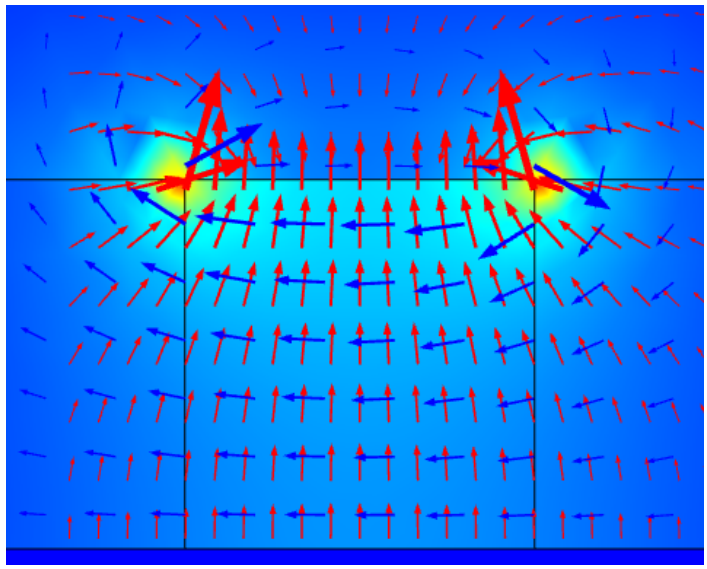

Fig. 5: 2D simulation of the lumped port 1 at $19 \mathrm{GHz}$. The electric field distribution is shown by the red arrows, the magnetic field distribution by the blue arrows.

matching around $19 \mathrm{GHz}$ can be achieved. The optimization of the matching is shown in Fig. 6. The best matching can be obtained at $t_{2}=1.7 \mathrm{~mm}$. However, detecting reflected signals below $-60 \mathrm{~dB}$ is very challenging, thus a taper-width $t_{2}$ of $1.625 \mathrm{~mm}$ has been chosen. Finally, matching of $-59.2 \mathrm{~dB}$ at $19.037 \mathrm{GHz}$ is achieved. The electric field distribution is shown in Fig. 7. The wave travels into the water. The colour scaling

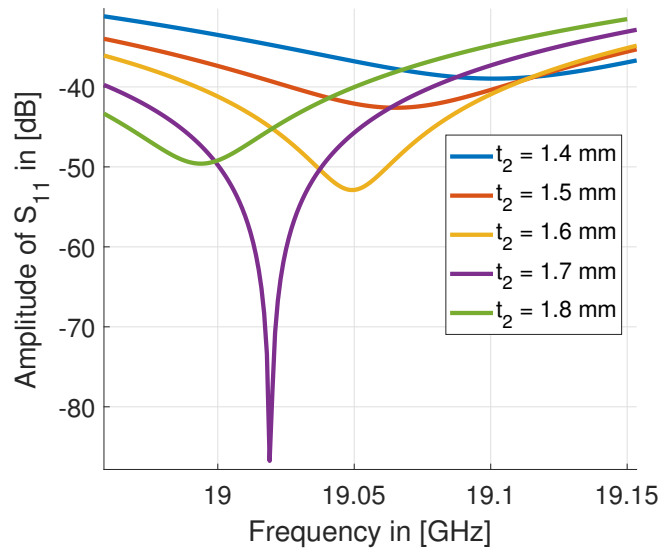

Fig. 6: The optimized taper-width is reached by varying the design parameter $t_{2}$. Optimal matching is achieved at $t_{2}=1.7 \mathrm{~mm}$.

Cross Section of the Absolute Value of the Electrical Field in $\mathrm{V} / \mathrm{m}$
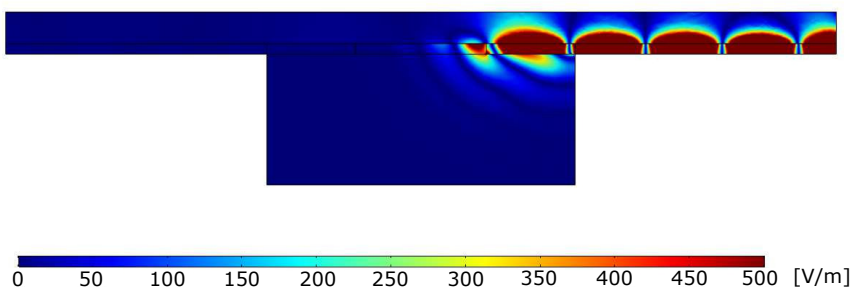

Fig. 7: The electric field distribution in the microstripline and its penetration in the water-tank.

of the field strength is adjusted in order to make low field strengths visible.

For each glucose-step the phase and absolute value of the transmission parameter $S_{21}$ and the reflection parameter $S_{11}$ are evaluated. Fig. 8 shows $S_{11}$ for a variation of glucoseconcentration. Regarding the reflection, $110 \mathrm{mg} / \mathrm{dl}$ correlates to an approximate $2 \mathrm{MHz}$ shift in frequency and $1.4 \mathrm{~dB}$ in absolute value. With increasing glucose concentration, the $3 \mathrm{~dB}$-bandwidth decreases since the dielectric losses also decrease (4). Both, the real and imaginary part of the relative permittivity influence the $3 \mathrm{~dB}$-bandwidth and the frequency shift in $S_{11}$. Fig. 9 shows the phase of $S_{11}$ for the different glucose concentration. The phase-differences are plotted in Fig. 10 and 11, considering $0 \mathrm{mg} / \mathrm{dl}$ glucose concentration as a reference. Around $19.037 \mathrm{GHz}$, one can observe the highest phase-difference. The sensitivity of the sensor is almost constant at $2{ }^{\circ}$ per $10 \mathrm{mg} / \mathrm{dl}$ and $10^{\circ}$ per $50 \mathrm{mg} / \mathrm{dl}$, respectively (Fig. 11). The constant phase-difference can be explained by the linear relation between the glucose concentration and the 


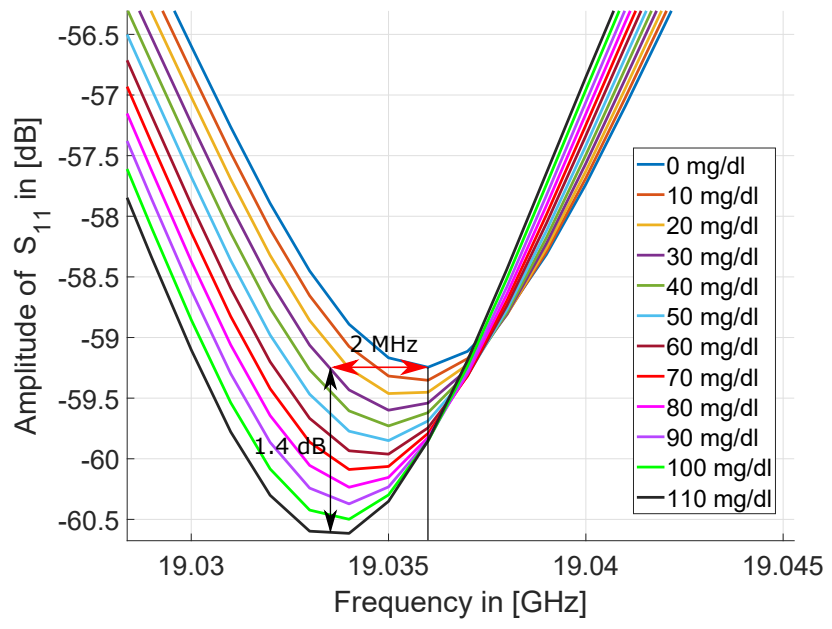

Fig. 8: Amplitude of $S_{11}$ versus frequency. The amplitude is decreased and shifted towards lower frequencies by increasing the glucose concentration (taper-width $t_{2}=1.625 \mathrm{~mm}$ ).

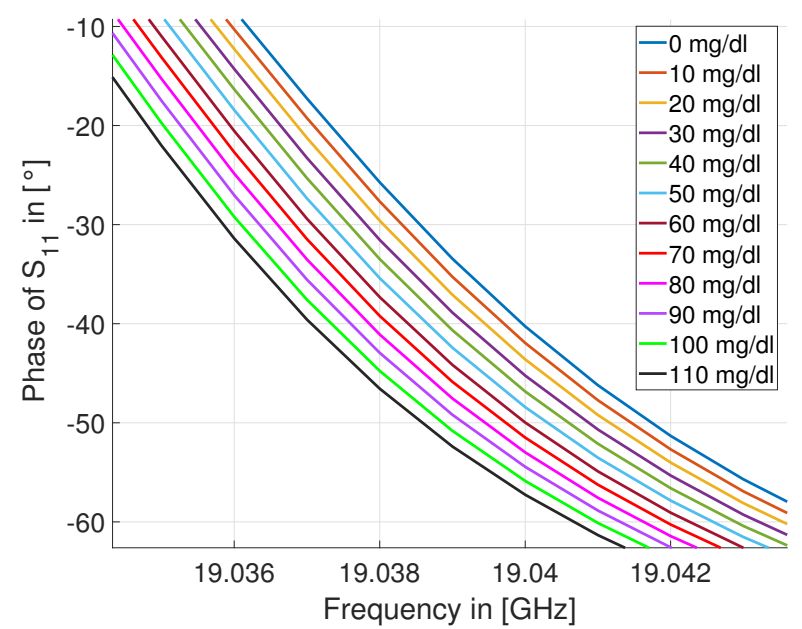

Fig. 9: Phase of $S_{11}$ versus frequency. The absolute value of the phase is increased and shifted towards the lower frequency by increasing the glucose concentration (taper-width $\left.t_{2}=1.625 \mathrm{~mm}\right)$.

viscosity [18]. Thus, there is a linear shift in the relaxation frequency, since the Debye model suggests a linear relation between glucose concentration and relaxation frequency for a small concentration like it is the case in human blood.

Fig. 12 shows the amplitude of $S_{21}$. A step of $10 \mathrm{mg} / \mathrm{dl}$ glucose concentration causes a variation of less than $10^{-2} \mathrm{~dB}$. With increasing glucose concentration the amplitude of $S_{21}$ increases. This coherence is described by (1) and (2). The phase of $S_{21}$ is shown in Fig. 13. The phase-difference is about $0.035^{\circ}$ on average as Fig. 14 suggests. Table I gives an overview of how the matching of $S_{11}$ impacts the phase-differences. The better the matching, the higher is the phase difference. This holds true for the reflection. For the

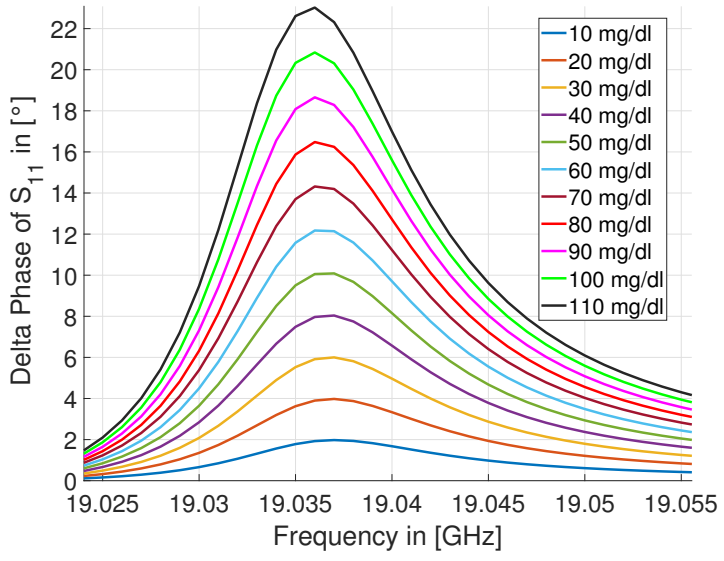

Fig. 10: Phase-differences of $S_{11}$ for various glucose concentration to the reference of $0 \mathrm{mg} / \mathrm{dl}$. At $19.037 \mathrm{GHz}$, there is the maximum phase-difference of approximately $2^{\circ}$ per $10 \mathrm{mg} / \mathrm{dl}$ glucose concentration.

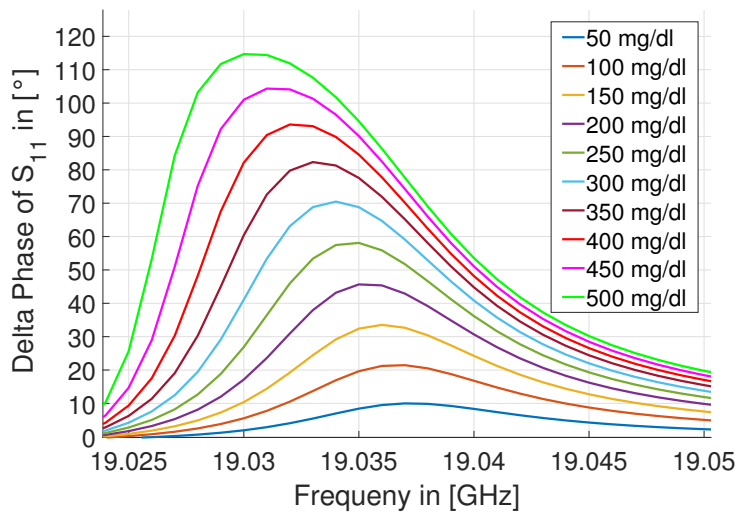

Fig. 11: Phase-differences of $S_{11}$ for high glucose concentration to the reference of $0 \mathrm{mg} / \mathrm{dl}$. Around $19.035 \mathrm{GHz}$, there is the maximum phase-difference of approximately $10^{\circ}$ per $50 \mathrm{mg} / \mathrm{dl}$ glucose concentration.

transmission however, there is rather no impact. The phasedifference of the transmission stays almost constant.

\section{DISCUSSION}

The results expose that the phase of the scattering parameters is more sensitive, than their amplitude. This is consistent with the earlier results published in [19]. There is no frequency shift visible in the transmission parameter $S_{21}$. This can be explained with the low slope of $\varepsilon_{\mathrm{r}}^{\prime \prime}(\omega)$ around the relaxation frequency. Within a bandwidth of $5.5 \mathrm{GHz}$, there is only approximately a change of 0.35 in $\varepsilon_{\mathrm{r}}^{\prime \prime}(\omega)$ (Fig. 15). This means that the change of $S_{21}$ is small as well. A broadband matching would be needed, if the absolute value of the transmission should be evaluated. This will evoke a ripple in the transmission, which can be proven by the Bode-Fano Criterion [20]. In the end, the dip in $S_{21}$ will be shaded by the ripple. 


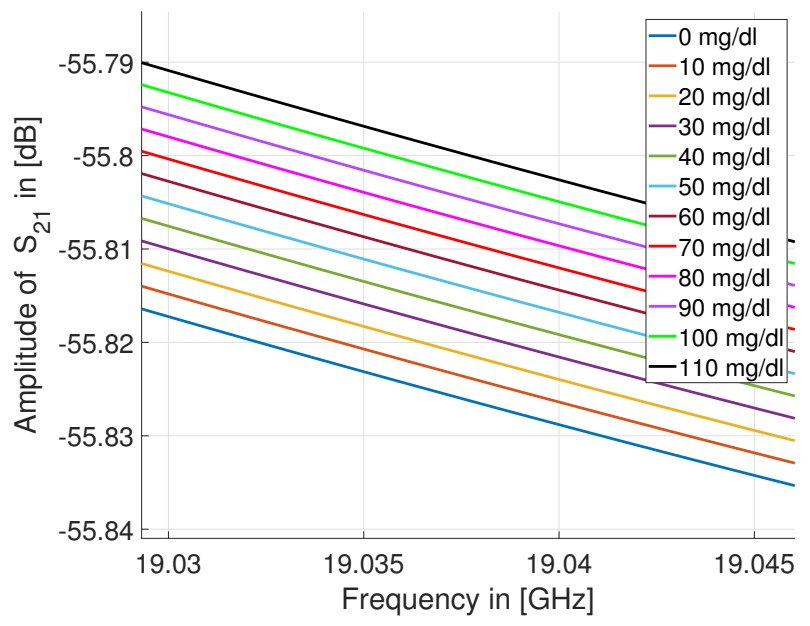

Fig. 12: Amplitude of $S_{21}$ versus frequency. The amplitude rises by increasing the glucose concentration (taper-width $\left.t_{2}=1.625 \mathrm{~mm}\right)$.

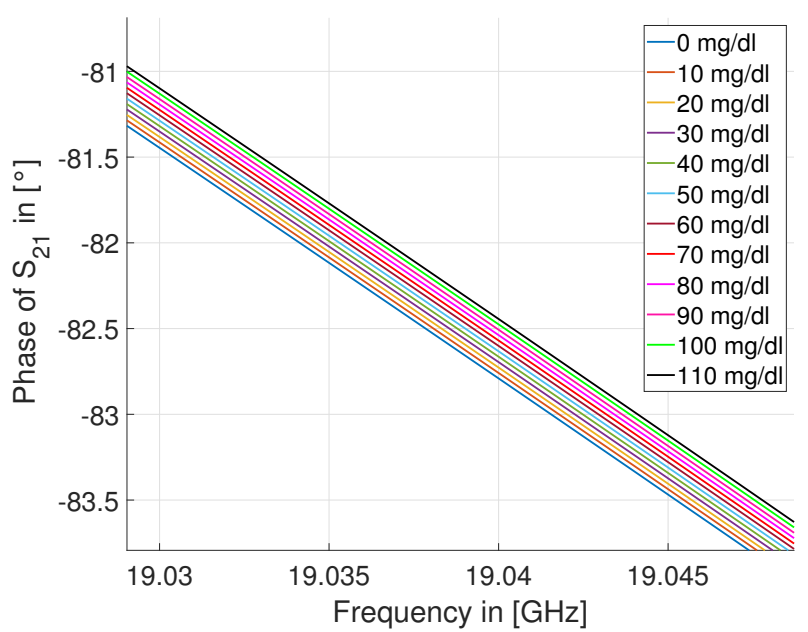

Fig. 13: Phase of $S_{21}$ versus frequency. The phase is decreased by increasing the glucose concentration (taper-width $\left.t_{2}=1.625 \mathrm{~mm}\right)$.

The findings also prove that with this setup, the phase of the reflection is more sensitive than the phase of the transmission. This allows to reduce the sensor to an one-port network. The amount of phase-difference per $10 \mathrm{mg} / \mathrm{dl}$ depends on the matching of $S_{11}$, which can be adjusted with the tapergeometry. However, the phase of the transmission is not affected by this. The resolution of other non-invasive glucose sensors is always worse than $10 \mathrm{mg} / \mathrm{dl}$. The best resolution of a microwave based non-invasive sensor, which was reached up to the current date, is $30 \mathrm{mg} / \mathrm{dl}$ [5]. However, the results of the proposed simulations show that a phase-difference of $2^{\circ}$ corresponds to a $10 \mathrm{mg} / \mathrm{dl}$ glucose concentration. The challenge will be the design of a read-out circuit for detecting the phase of reflected signals around $-60 \mathrm{~dB}$.

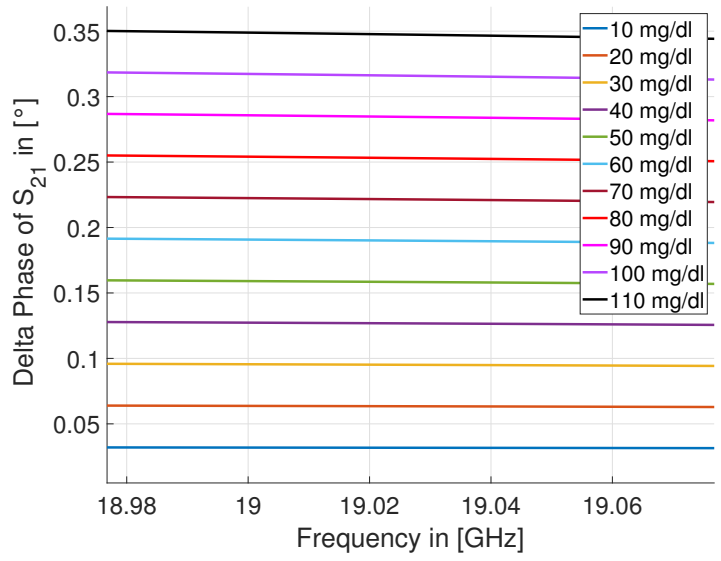

Fig. 14: Phase-differences of $S_{21}$ for various glucose concentration to the reference of $0 \mathrm{mg} / \mathrm{dl}$. The phase-difference is almost constant over the frequency $0.035^{\circ}$ per $10 \mathrm{mg} / \mathrm{dl}$ glucose concentration.

TABLE I: Average phase-differences for different $S_{11}$.

\begin{tabular}{cccc}
\hline$S_{11}[\mathrm{~dB}]$ & $t_{2}[\mathrm{~mm}]$ & $\begin{array}{c}\text { Reflection } \\
\Delta \phi_{\mathrm{S}_{11}}\end{array}$ & $\begin{array}{c}\text { Transmission } \\
\Delta \phi_{\mathrm{S}_{21}}\end{array}$ \\
\hline-38.9 & 1.4 & 0.18 & 0.06 \\
-42.6 & 1.5 & 0.28 & 0.05 \\
-52.9 & 1.6 & 0.95 & 0.05 \\
-59.2 & 1.625 & 1.98 & 0.05 \\
-86.5 & 1.7 & 7.5 & 0.05 \\
\hline
\end{tabular}

\section{Implementation Challenges}

The first step in order to validate the results in a practical measurement is to fabricate a testbed in the laboratory with discrete components. However, good matching is crucial to guarantee a high phase-difference. The scattering parameter $S_{11}$ depends on the geometry of the sensor and the permittivity of the substrate. The design dielectric constant of the substrate is only an approximate value. Therefore, an impedancetuner has to be added in the setup to correct mismatching. Additional, highly phase stable cables have to be used to guarantee accurate phase-measurements. The measuring of the phase is narrow-banded. Even a high-end vector-networkanalyzer (VNA) as the $R \& S^{\circledR}$ ZNA 26 is not a proper choice since the implemented coupler has a too low directivity. A reflection measurement below $-50 \mathrm{~dB}$ would result in extreme high requirements for a VNA. Therefore, a narrow-banded setup, as introduced in [21], would be a better option. With the presented read-out circuit, a resolution of the reflection coefficient phase of $0.4^{\circ}$ was reached.

Moreover, the substrate should have a low moistureabsorption and a passivation layer should be added to the substrate as it has direct contact to the water.

\section{CONClusion}

In this paper, a non-invasive glucose sensor at $19 \mathrm{GHz}$ has been proposed. A two-port MSL with a tapered ground plane 


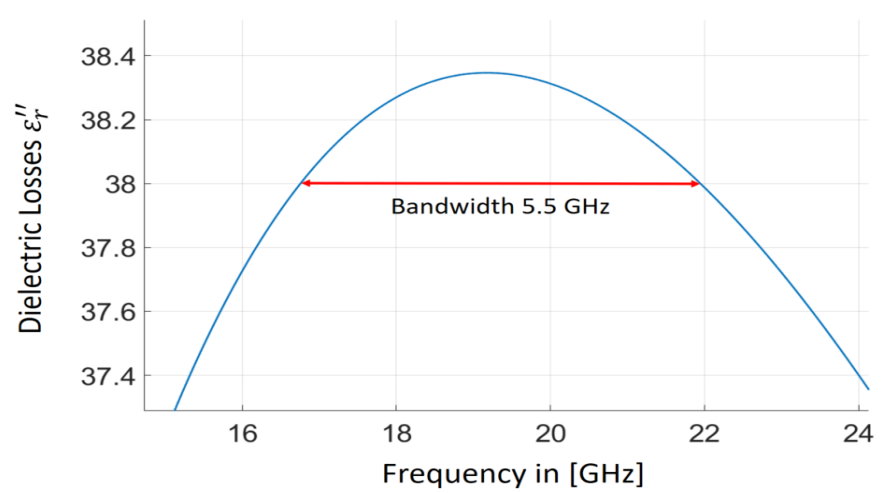

Fig. 15: Graph of the dielectric losses suggested by Debye's model.

was used to design the sensor. By evaluating the phase of $S_{11}$ for the various glucose concentration and comparing it with the reference phase of $0 \mathrm{mg} / \mathrm{dl}$, a $2^{\circ}$ phase change, corresponding to the $10 \mathrm{mg} / \mathrm{dl}$ glucose concentration, was achieved. The simulation results indicate that the phase-change of the reflected wave from the interface depends on the matching, and no significant phase-change was observed at port 2. Finally, the challenges of a practical implementation were specified. Further research is focused on practical measurements to validate the simulation results.

\section{REFERENCES}

[1] N. H. Cho, J. Shaw, S. Karuranga, Y. Huang, J. D. da Rocha Fernandes, A. Ohlrogge, and B. Malanda, "Idf diabetes atlas: Global estimates of diabetes prevalence for 2017 and projections for 2045," Diabetes Research and Clinical Practice, vol. 138, Feb. 2018.

[2] G. Eigner, P. I. Sas, and L. Kovács, "Continuous glucose monitoring systems in the service of artificial pancreas," in IEEE 9th IEEE International Symposium on Applied Computational Intelligence and Informatics (SACI), 2014, pp. 117-122.

[3] W. Villena Gonzales, A. Mobashsher, and A. Abbosh, "The progress of glucose monitoring-a review of invasive to minimally and non-invasive techniques, devices and sensors," Sensors, vol. 19, p. 800, Feb. 2019.

[4] T. Yilmaz, R. Foster, and Y. Hao, "Radio-frequency and microwave techniques for non-invasive measurement of blood glucose levels," MDPI Diagnostics, vol. 9, p. 6, Jan. 2019.

[5] M. Islam, M. Hoque, A. Almutairi, and N. Amin, "Left-handed metamaterial-inspired unit cell for s-band glucose sensing application," Sensors, vol. 19, p. 169, Jan. 2019.

[6] H. Choi, J. Nylon, S. Luzio, J. Beutler, and A. Porch, "Design of continuous non-invasive blood glucose monitoring sensor based on a microwave split ring resonator," in IEEE MTT-S International Microwave Workshop Series on RF and Wireless Technologies for Biomedical and Healthcare Applications (IMWS-Bio2014), 2014, pp. 1-3.

[7] D. Mondal, N. K. Tiwari, and M. J. Akhtar, "Microwave assisted noninvasive microfluidic biosensor for monitoring glucose concentration," in IEEE SENSORS, 2018, pp. 1-4.

[8] H. Choi, J. Naylon, S. Luzio, J. Beutler, J. Birchall, C. Martin, and A. Porch, "Design and in vitro interference test of microwave noninvasive blood glucose monitoring sensor," IEEE Transactions on Microwave Theory and Techniques, vol. 63, no. 10, pp. 3016-3025, 2015.

[9] T. Yilmaz, R. Foster, and Y. Hao, "Patch resonator for non-invasive detection of dielectric property changes in biological tissues," in Proceedings of the 2012 IEEE International Symposium on Antennas and Propagation, 2012, pp. 1-2.

[10] H. Cano-Garcia, I. Gouzouasis, I. Sotiriou, S. Saha, G. Palikaras, P. Kosmas, and E. Kallos, "Reflection and transmission measurements using $60 \mathrm{ghz}$ patch antennas in the presence of animal tissue for noninvasive glucose sensing," in 10th European Conference on Antennas and Propagation (EuCAP), 2016, pp. 1-3.

[11] M. Hofmann, "Six-port-based microwave spectroscopy of dielectric materials for non-invasive ism applications: Sechstor-basierte mikrowellenspektroskopie dielektrischer materialien für nicht-invasive ism anwendungen," Ph.D. dissertation, FAU University Press, Erlangen, 2015. [Online]. Available: https://nbn-resolving.org/urn:nbn:de:bvb:29opus4-67197

[12] B. Greenebaum, Bioengineering and Biophysical Aspects of Electromagnetic Fields, Nov. 2018

[13] W. Ebeling, "Book review: Physical chemistry of electrolyte solutions. modern aspects. topics in physical chemistry 5, by j. m. g. barthel, h. krienke, and w. kunz," Journal of Solution Chemistry, vol. 29, no. 2, pp. 199-200, Feb. 2000. [Online]. Available: https://doi.org/10.1023/A:1005177316075

[14] P. J. W. Debye, Polar molecules. Chemical Catalog Company, 1929.

[15] V. Turgul and I. Kale, "Characterization of the complex permittivity of glucose/water solutions for noninvasive rf/microwave blood glucose sensing," in IEEE International Instrumentation and Measurement Technology Conference Proceedings, 2016, pp. 1-5.

[16] M. Hofmann, F. Trenz, R. Weigel, G. Fischer, and D. Kissinger, "A microwave sensing system for aqueous concentration measurements based on a microwave reflectometer," in IEEE/MTT-S International Microwave Symposium Digest, 2012, pp. 1-3.

[17] W. L. Clarke, D. C. Cox, L. Gonder-Frederick, W. Carter, and S. L. Pohl, "Evaluating clinical accuracy of systems for self-monitoring of blood glucose," Diabetes care, vol. 10, pp. 622-8, Sep. 1987.

[18] L. A. Bulavin, Y. F. Zabashta, A. M. Khlopov, and A. V. Khorol'skii, "Molecular mechanism of the viscosity of aqueous glucose solutions," Russian Journal of Physical Chemistry A, vol. 91, no. 1, pp. 89-93, Jan. 2017. [Online]. Available: https://doi.org/10.1134/S0036024416120062

[19] M. Hofmann, M. Bloss, R. Weigel, G. Fischer, and D. Kissinger, "Noninvasive glucose monitoring using open electromagnetic waveguides," in 42nd European Microwave Conference, 2012, pp. 546-549.

[20] R. M. Fano, "Theoretical limitations on the broadband matching of arbitrary impedances," Journal of the Franklin Institute, vol. 249, no. 1, pp. 57-83, Jan. 1950. [Online]. Available: http://www.sciencedirect.com/science/article/pii/0016003250900068

[21] B. Laemmle, K. Schmalz, C. Scheytt, R. Weigel, and D. Kissinger, "An integrated $125 \mathrm{ghz}$ sensor with read-out circuit for permittivity measurement of liquids," in IEEE/MTT-S International Microwave Symposium Digest, 2012, pp. 1-3. 